\title{
Optimal Color Stability for White Organic Light-Emitting Diode (WOLED) by Using Multiple-Ultra-Thin Layers (MUTL)
}

\author{
Kan-Lin Chen, ${ }^{1}$ Chien-Jung Huang, ${ }^{2}$ Wen-Ray Chen, ${ }^{3}$ Fong-Yi Lin, ${ }^{2}$ \\ Teen-Hang Meen, ${ }^{3}$ and Chih-Chieh Kang ${ }^{4}$ \\ ${ }^{1}$ Department of Electronic Engineering, Fortune Institute of Technology, Kaohsiung 83160, Taiwan \\ ${ }^{2}$ Department of Applied Physics, National University of Kaohsiung, Kaohsiung 81148, Taiwan \\ ${ }^{3}$ Department of Electronic Engineering, National Formosa University, Hu-Wei, Yunlin 63201, Taiwan \\ ${ }^{4}$ Department of Electro-Optical Engineering, Southern Taiwan University of Science and Technology, Tainan 71005, Taiwan
}

Correspondence should be addressed to Kan-Lin Chen; klchen@fotech.edu.tw and Chien-Jung Huang; chien@nuk.edu.tw

Received 15 September 2013; Accepted 3 October 2013

Academic Editor: Liang-Wen Ji

Copyright (c) 2013 Kan-Lin Chen et al. This is an open access article distributed under the Creative Commons Attribution License, which permits unrestricted use, distribution, and reproduction in any medium, provided the original work is properly cited.

\begin{abstract}
The work demonstrates the improvement of color stability for white organic light-emitting diode (WOLED). The devices were prepared by vacuum deposition on ITO-glass substrates. These guest materials of 5,6,11,12-tetraphenylnaphthacene (Rubrene) were deposited in 4,4'-bis(2,2-diphenyl vinyl)-1,1'-biphenyl (DPVBi), resulting in an emitting layer. Experimental results reveal that the properties in the multiple-ultra-thin layer (MUTL) are better than those of the emitting layer with a single guest material, reaching the commercial white-light wavelength requirement of 400-700 $\mathrm{nm}$. The function of the MUTL is as the light-emitting and trapping layer. The results show that the MUTL has excellent carrier capture effect, leading to high color stability of the device at various applied voltages. The Commissions Internationale De L'Eclairage (CIE) coordinate of this device at 3 7 V is few displacement and shows a very slight variation of $(0.016,0.009)$. The CIE coordinates at a maximal luminance of $9980 \mathrm{~cd} / \mathrm{m}^{2}$ are $(0.34,0.33)$.
\end{abstract}

\section{Introduction}

Since Tang and Vanslyke first reported on a multilayer organic light-emitting diode (OLED), OLEDs have been widely investigated for their potential application in flat-panel displays [1]. OLED has attracted attention owing to its advantageous emission over a wide visible range and its application in flatpanel displays that are driven at low voltage [2-4]. Furthermore, the development of white organic light-emitting diode (WOLED) with high brightness and good color purity is necessary, because these devices can be employed not only as illumination light source but also as backlight source candidate for next generation flat-panel displays. However, the WOLED lighting technology has been extensively studied. To achieve white emission from OLEDs, various several methods have been tried to obtain optimal WOLEDs, for example, using two complementary colors (blue and yellow), multilayer stack of three primary colors (red, green, and blue) and two or three colors of the dye doped into single host material [5-7]. In the co-deposition process, accurately controlling the evaporation rate and the concentration of the two or more materials is not easy, resulting in its poor performance. However, non-doped technique can accurately control to avoid the above problems in the fabrication processes.

In this work, we use nondoped method to fabricate WOLEDs with a multiple-ultra-thin layer (MUTL) structure. The 5,6,11,12-tetraphenylnaphthacene (Rubrene) material was used as yellow light sources in MUTL structure. In addition, the quantum efficiency of the Rubrene can be close to $100 \%$ [8-11]. And then the material of 4,40-bis (2,20-diphenyl vinyl)-1,10-biphenyl (DPVBi) is well-known blue fluorescent material. However, DPVBi is not only emitting but also transfering the incomplete energy from DPVBi to the Rubrene. So far the study of fluorescence WOLED (FWOLED) based on the non-doped multiple-ultra-thin layer (MUTL) has not yet 


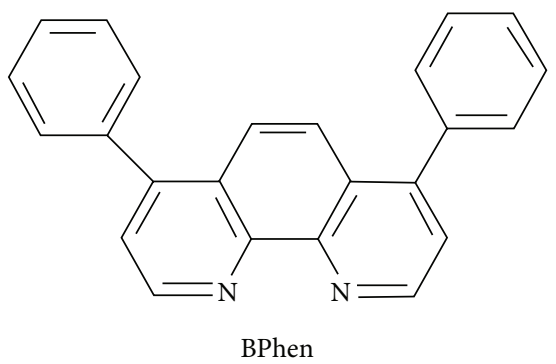<smiles>C(=C(c1ccccc1)c1ccc(-c2ccccc2)cc1)c1ccccc1</smiles>
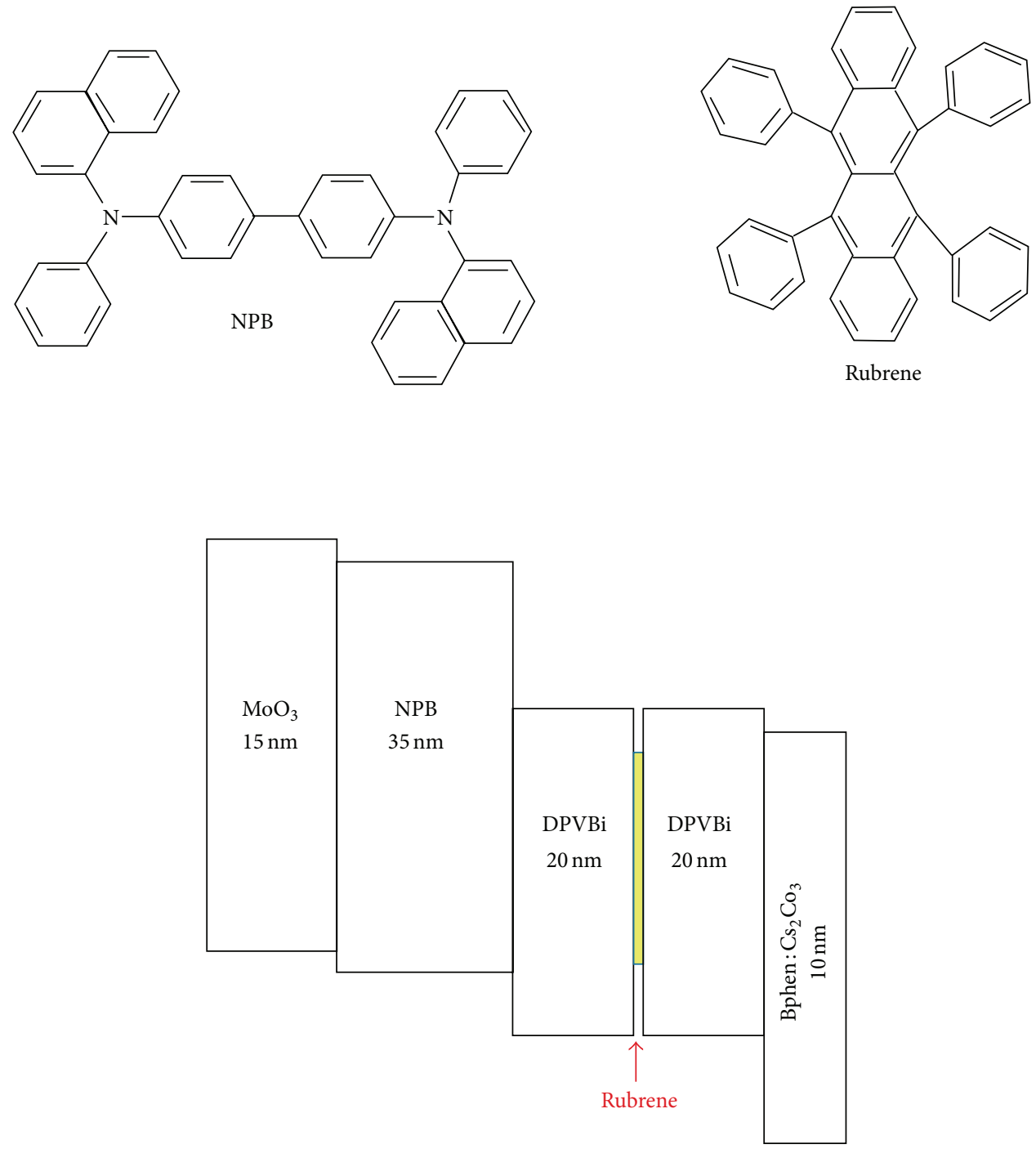

FIGURE 1: The chemical structures of organic materials and the structures of the device in the energy band diagrams of the multilayer (Rubrene $=0.1,0.2$ and $0.3 \mathrm{~nm}$ ) for WOLEDs.

been established. Therefore, this paper concerns a simple process for the non-doped FWOLEDs with a MUTL structure, ensuring that the improvement of FWOLEDs performance was highly efficient due to the good capture efficiency of charge carrier for the guest material. Besides, a detailed investigation of the transmission mechanism for the MUTL structure on the electroluminescence (EL) and the color stability of FWOLEDs is presented.

\section{Experimental}

Figure 1 shows the chemical structures of organic materials and the structures of the device that are used in this study. Indium tin oxide (ITO) coated glass with a sheet resistance of approximately $15 \Omega /$ sq was consecutively cleaned in ultrasonic bath containing detergent water, acetone, ethanol and deionized (DI) water for $20 \mathrm{~min}$ each and then dried with 


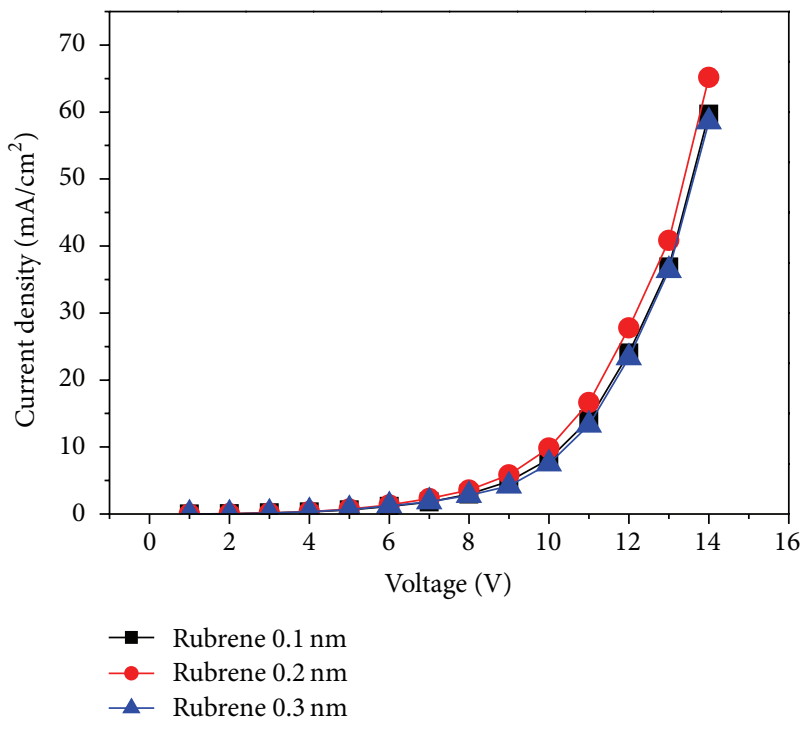

Figure 2: The current density-voltage (J-V) characteristics of the devices with various thicknesses of Rubrene layer.

a nitrogen $\left(\mathrm{N}_{2}\right)$ flow. All organic layers were sequentially evaporated onto the ITO coated-glass substrate at highvacuum $\left(1 \times 10^{-6}\right.$ Torr $)$ thermal evaporation. Thermal deposition rates for organic materials, inorganic materials, and Al were about $1 \AA / \mathrm{sec}, 1 \AA / \mathrm{sec}$, and $10 \AA / \mathrm{sec}$, respectively. The evaporation rate and thickness of the thin films were controlled using a quartz-crystal monitor system (Sigma, SID-142) in this work.

The active area of the device was $0.6 \mathrm{~cm}^{2}$. To measure the properties of the device, a voltage was applied by using a Keithley 2400 programmable voltage-current source (Keithley SourceMeter 2400, USA). EL spectra and Commissions Internationale De L'Eclairage (CIE) coordination of the devices were measured by PR655 spectra scan spectrometer (Kollmorgen Instrument PR655, USA). All measurements were made at room temperature without encapsulating the devices.

\section{Results and Discussion}

In order to fabricate WOLED, we first attempted to obtain the optimizing thicknesses of Rubrene. Generally, yellow light is required as one of the two-color complementary to obtain white light. The structure of the device in energy band diagrams of the multilayer for WOLEDs is shown in Figure 1. The thicknesses of Rubrene layer change from 0.1 to $0.3 \mathrm{~nm}$ at fixing other organic layers. Figure 2 shows the current density-voltage (J-V) characteristics of the devices with various thicknesses of Rubrene layer. The current density of the devices at $14 \mathrm{~V}$, whose thicknesses of Rubrene layer are $0.1 \sim 0.3 \mathrm{~nm}$ are $59.6,65.1$ and $58.5 \mathrm{~mA} / \mathrm{cm}^{2}$, respectively. It can be observed that the device whose thickness of Rubrene layer is $0.2 \mathrm{~nm}$ has the best J-V characteristics as compared with other devices. Figure 3 shows the luminance-voltage (LV) curves of the devices with various thicknesses of Rubrene



FIgURE 3: The luminance-voltage curves of the devices with various thicknesses of Rubrene layer.

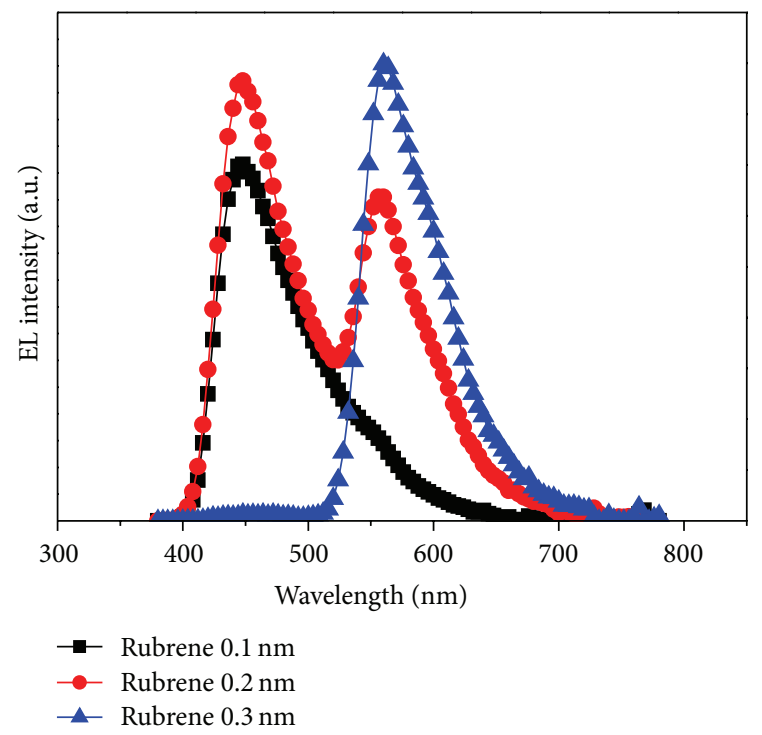

FIGURE 4: The electroluminescence spectra of the devices with various thicknesses of Rubrene layer at $14 \mathrm{~V}$.

layer. The maximum luminance of devices is 1476,1806 , and $1566 \mathrm{~cd} / \mathrm{m}^{2}$, respectively. The maximum luminance is $1806 \mathrm{~cd} / \mathrm{m}^{2}$ at $14 \mathrm{~V}$ and the CIE coordinate is $(0.270,0.286)$ when Rubrene layer is $0.2 \mathrm{~nm}$. Besides, the luminance of the device with Rubrene layer of $0.2 \mathrm{~nm}$ is higher than other devices at the same voltage. The phenomenon tells us that proper thickness of Rubrene layer results in better luminance for WOLED. The device with Rubrene layer of $0.2 \mathrm{~nm}$ is ideal because of the best luminance in other devices at the same applying voltage. However, the device with proper thickness of Rubrene layer can produce a high luminance. Figure 4 shows normalized electroluminescence spectra of the devices at $14 \mathrm{~V}$ with various thicknesses of 


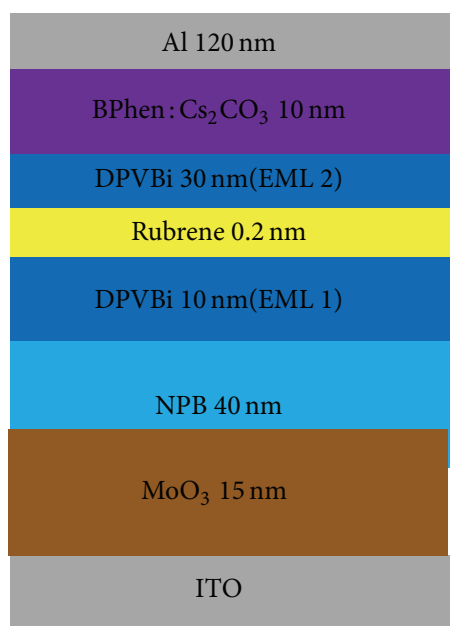

Device A



Device B

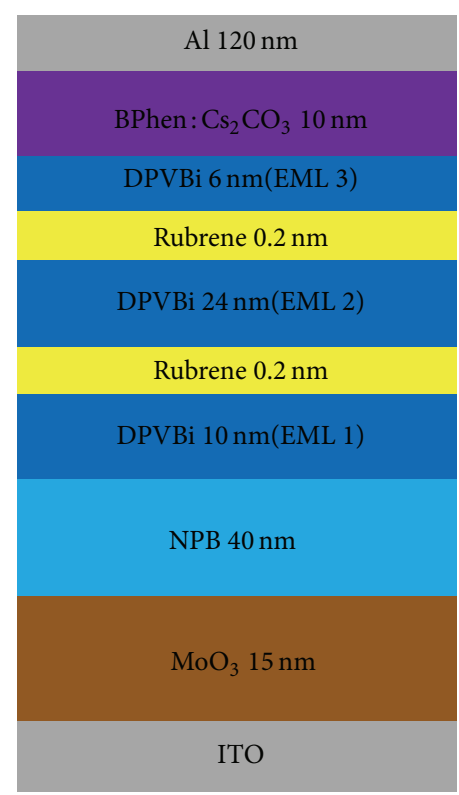

Device C

FIGURE 5: The structure of WOLEDs.

Rubrene layer at $0.1,0.2$ and $0.3 \mathrm{~nm}$, respectively. It is well known that the peak wavelengths of the DPVBi layer and the Rubrene layer were $436 \mathrm{~nm}$ and $556 \mathrm{~nm}$, respectively $[12,13]$. It can be observed that light emission of three devices is composed of yellow light emission and blue light emission. In addition, it can be found that the devices whose thicknesses of Rubrene layer are 0.1 and $0.3 \mathrm{~nm}$ all have an EL peak without the balances of blue and yellow emission intensity. The device with Rubrene layer of $0.2 \mathrm{~nm}$ has a CIE coordinate of $(0.270,0.286)$ which lies in about the blue color zone. However, the device with $0.2 \mathrm{~nm}$ Rubrene layer at $14 \mathrm{~V}$ has better luminance of $1806 \mathrm{~cd} / \mathrm{m}^{2}$. In this study, the white light emission of device consists of the blue emission layer and the yellow emission layer. The yellow emission of the device was caused by the Rubrene layer, which was inserted in the light-emitting layer of DPVBi to form the structure of DPVBi (10 nm EML1)/Rubrene $(0.2 \mathrm{~nm}) / \mathrm{DPVBi}(30 \mathrm{~nm}$ EML2). If the intensity of blue emission was higher than that of the yellow emission, then the CIE coordinates of the device were unstable. This is due to the fact that the Rubtene layer in the device cannot trap enough electrons and holes to generate exciton. Therefore, optimum complementary color of the blue and the yellow emission intensity was not achieved. According to the results obtained above, the best recombination zone was in the EML2 of the device. To achieve an objective of the optimal color stability, three structures of devices, as shown in Figure 5, are promoted and fabricated as follows.

(a) ITO/Molybdenum trioxide $\left(\mathrm{MoO}_{3} ; 15 \mathrm{~nm}\right) / \mathrm{N}, \mathrm{N} 0-$ bis-(1-naphthyl)-N,N0-biphenyl-1,10-biphenyl-4,40diamine $(\mathrm{NPB} ; 40 \mathrm{~nm}) / \mathrm{DPVBi} \quad(10 \mathrm{~nm}) /$ Rubrene
$(0.2 \mathrm{~nm}) / \mathrm{DPVBi}(30 \mathrm{~nm}) / 4,7-$ Diphenyl-1,10-phenanthroline: cesium carbonate (BPhen: $\mathrm{Cs}_{2} \mathrm{Co}_{3}=4: 1$; $10 \mathrm{~nm}$ )/Aluminum (Al; $120 \mathrm{~nm})$.

(b) $\mathrm{ITO} / \mathrm{MoO}_{3}(15 \mathrm{~nm}) / \mathrm{NPB}(40 \mathrm{~nm}) / \mathrm{DPVBi}(34 \mathrm{~nm}) /$ Rubrene $(0.2 \mathrm{~nm}) / \mathrm{DPVBi}(6 \mathrm{~nm}) / \mathrm{BPhen}: \mathrm{Cs}_{2} \mathrm{Co}_{3}=$ $4: 1(10 \mathrm{~nm}) / \mathrm{Al}(120 \mathrm{~nm})$.

(c) $\mathrm{ITO} / \mathrm{MoO}_{3}(15 \mathrm{~nm}) / \mathrm{NPB}(40 \mathrm{~nm}) / \mathrm{DPVBi}(10 \mathrm{~nm}) /$ Rubrene $(0.2 \mathrm{~nm}) / \mathrm{DPVBi}(24 \mathrm{~nm}) /$ Rubrene $(0.2 \mathrm{~nm}) /$ DPVBi $(6 \mathrm{~nm}) / \mathrm{BPhen}: \mathrm{Cs}_{2} \mathrm{Co}_{3}=4: 1(10 \mathrm{~nm}) / \mathrm{Al}$ $(120 \mathrm{~nm})$.

The current density-voltage-luminance characteristics of devices $\mathrm{A} \sim \mathrm{C}$ are shown in Figures 6(a) and 6(b). The maximum current density of devices $\mathrm{A}, \mathrm{B}$, and $\mathrm{C}$ is $203 \mathrm{~mA} / \mathrm{cm}^{2}$, $298 \mathrm{~mA} / \mathrm{cm}^{2}$ and $355 \mathrm{~mA} / \mathrm{cm}^{2}$ at $7 \mathrm{~V}$, respectively. It can be seen that current density of device $\mathrm{C}$ exceeds the other two devices at the same voltage. This is the reason why device $\mathrm{C}$ has larger luminance. The maximum luminance of the devices A-C is $6010 \mathrm{~cd} / \mathrm{m}^{2}, 7768 \mathrm{~cd} / \mathrm{m}^{2}$, and $9980 \mathrm{~cd} / \mathrm{m}^{2}$, respectively. It can be explained that the holes were accumulated at the Rubrene/EML3 interface and that the electrons were accumulated at the BPhen: $\mathrm{Cs}_{2} \mathrm{Co}_{3}$ layer, indicating that the electric field can be formed in EML3. The electric field is favorable for electron injection into the emission layer. However, it is expected that the holes were trapped and confined in the Rubrene/EML3 interface and electron injection thus enhanced, resulting that an improved carrier balance was achieved.

EL spectra and CIE coordinates during the applied voltage of $3 \sim 7 \mathrm{~V}$ are shown in Figure 7. It can be found that there is a balance or complementary color between blue emission and yellow emission, as shown in Figure 7(a). Besides, a pure white light emission with CIE coordinates of $(0.331,0.332)$ is observed at the applied voltage of $5 \mathrm{~V}$. When the applied 


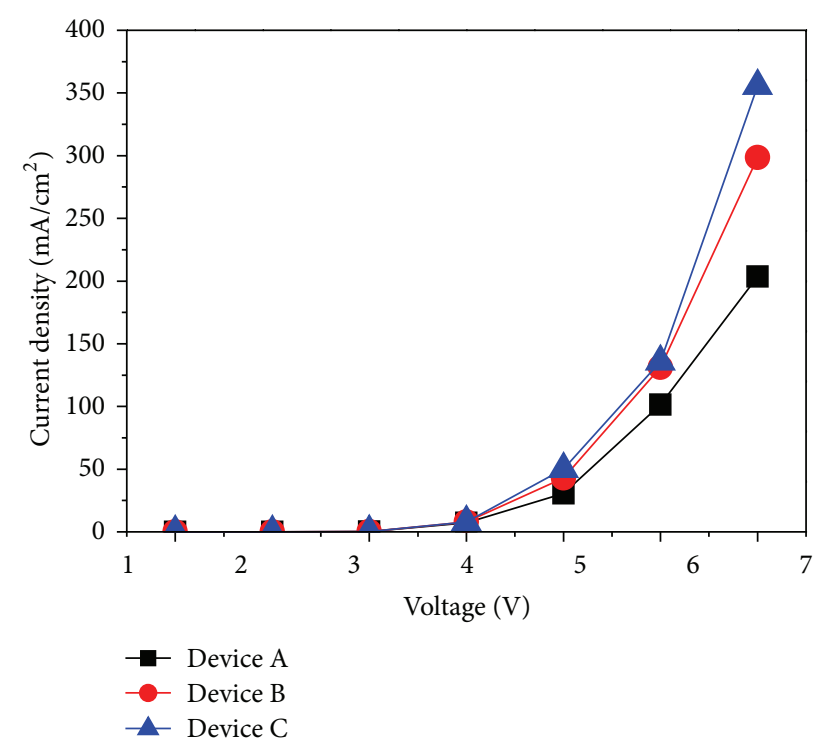

(a)

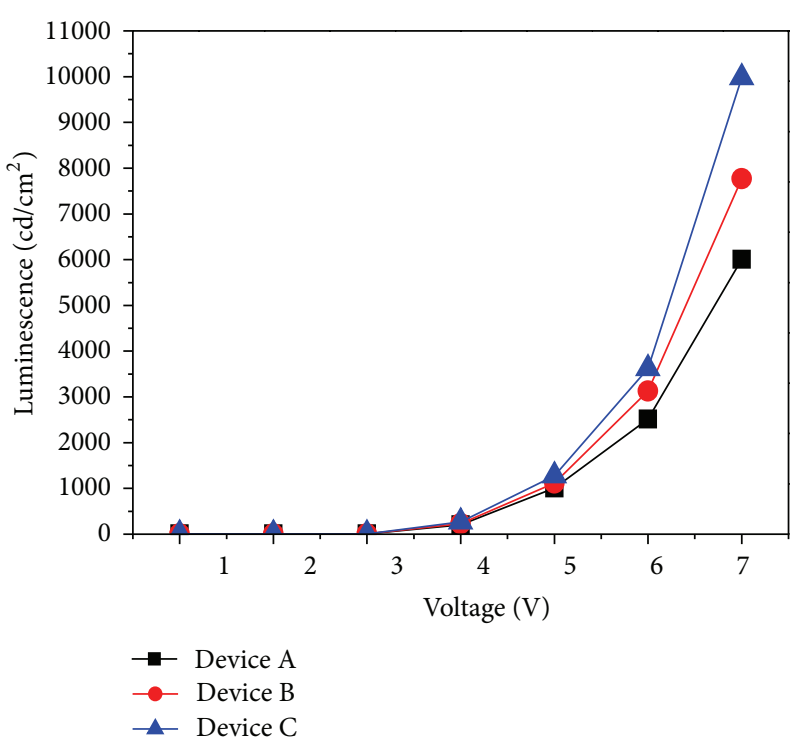

(b)

Figure 6: (a) The current density versus bias voltage (J-V) characteristics of devices A C. (b) The luminance versus voltage (L-V) characteristics of devices $\mathrm{A} \sim \mathrm{C}$.

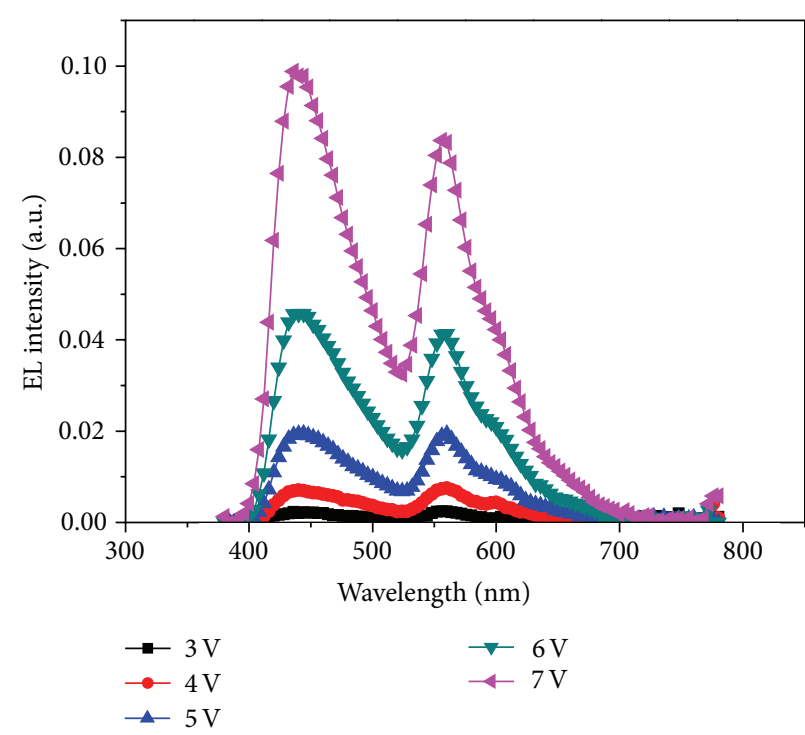

(a)

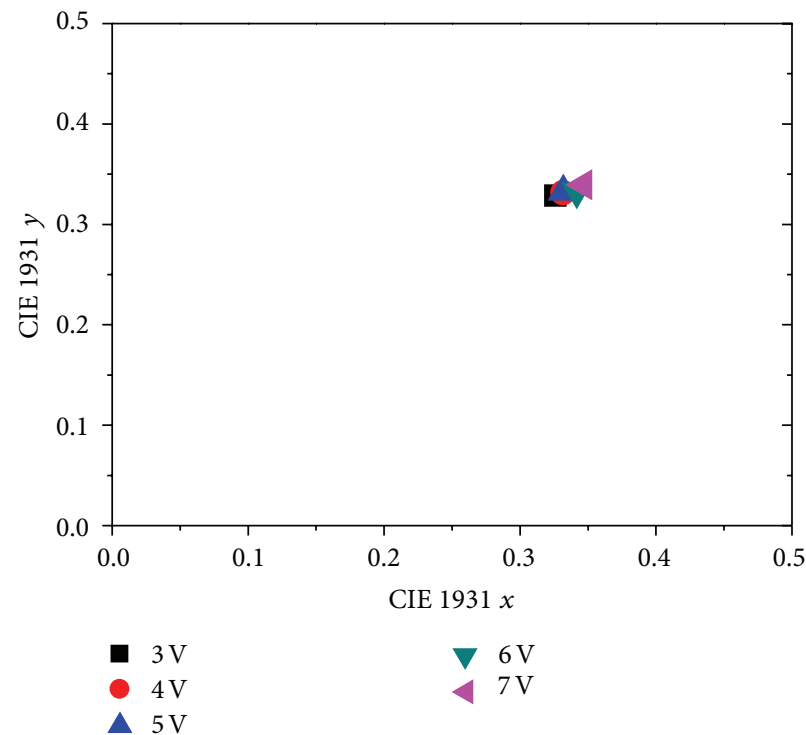

(b)

FIgure 7: (a) The EL spectra of device C at 3 7 V applied voltage. (b) The CIE coordinates of device C at 3 7 V applied voltage.

voltage increases from $6 \mathrm{~V}$ to $7 \mathrm{~V}$, the $\mathrm{CIE}$ coordinates were, respectively, $(0.341,0.332)$ and $(0.346,0.339)$, and showed a little change. This is owing to the fact that the intensity for the ratio of blue and yellow emission from EL spectra of Figure 7(a) is almost the same and about unit. Furthermore, this improvement in chromaticity can be attributed to the MUTL structure of the emission layer, resulting in a balance in the relative intensity of blue and yellow emission. Thus, by introducing a MUTL structure in the emission layer, the WOLED has more stable spectra characteristics than that of devices $\mathrm{A}$ and $\mathrm{B}$ with the increase of bias voltage.
This is due to the reason that the injection of holes and electrons was enhanced by the MUTL structure and that the MUTL structure can enhance charge carrier trapping in the EML1/Rubrene, EML2/Rubrene and Rubrene/EML3 interfaces. And then the enhanced carrier injection was because of electric fields induced by the accumulated carrier between interfaces of the MUTL. This result is similar to the result discussed in other literature [14-17]. In other words, the excitons formed from electrons and holes can widely distribute in the entire emission layer. As is expected, the excitons of Rubrene and DPVBi layers will increase and 
TABLE 1: Various characteristics of devices A-C.

\begin{tabular}{lccc}
\hline & Device A & Device B & Device C \\
\hline Turn-on voltage $(\mathrm{V})$ & 3.5 & 3.2 & 3 \\
Operating voltage $(\mathrm{V})$ & 4.1 & 3.9 & 3.5 \\
Maximum luminance $\left(\mathrm{cd} / \mathrm{m}^{2}\right)$ & 6010 & 7768 & 9980 \\
Maximum Current density $\left(\mathrm{cd} / \mathrm{m}^{2}\right)$ & 203 & 298 & 355 \\
\hline
\end{tabular}

reach balance, indicating that the MUTL can enhance color stability of CIE coordinates and chromaticity of pure white emission. However, the displacement of the CIE coordinates during the applied voltage of $3 \sim 7 \mathrm{~V}$ is almost null.

All the above data, can be summarized in Table 1. From Table 1, it is found that the MUTL structure can enhance color stability of CIE coordinates and chromaticity of pure white emission. Furthermore, these results show that a high luminance can be achieved with improved carrier balance in the emission zone. Besides, the operating voltage of device $\mathrm{C}$ is lower than that of other devices due to the presence of the MUTL structure, resulting in more electron injection and balance.

\section{Conclusions}

In summary, white light emission was achieved by inserting the MUTL structure in the emission layer of WOLED. Experimental results reveal that the properties in the multiple-ultrathin layer (MUTL) are better than those of the emitting layer with a single guest material. The carrier trapping of the MUTL structure can more effectively confine electrons and holes in the emission layer. Consequently, the excitons of Rubrene and DPVBi layers will increase and reach balance; that is, there is a balance or complementary color in blue emission and yellow emission, resulting in good color stability characteristics of WOLED at various applied voltages. A pure white emission CIE coordinates of $(0.331,0.332)$ can be obtained and show a little change at the applied voltage of $3 \sim 7 \mathrm{~V}$. It is demonstrated that EL emission is less dependent on the applied voltage. Thus, the carrier recombination zone must be properly controlled by limiting the exciton zone to prevent the mixing of colors. However, device with a MUTL structure increased carrier tapping probability to improve carrier recombination and luminous efficiency.

\section{Acknowledgment}

This work was partially supported by the National Science Council of the Republic of China under Contract no. NSC 102-2221-E-390-019-MY2.

\section{References}

[1] C. W. Tang and S. A. Vanslyke, "Organic electroluminescent diodes," Applied Physics Letters, vol. 51, no. 12, pp. 913-915, 1987.

[2] C. J. Huang, T. H. Meen, S. L. Wu, and C. C. Kang, "Improvement of color purity and electrical characteristics by co-doping method for flexible red-light organic light emitting devices," Displays, vol. 30, no. 4-5, pp. 164-169, 2009.
[3] Y. Sun, N. C. Giebink, H. Kanno, B. Ma, M. E. Thompson, and S. R. Forrest, "Management of singlet and triplet excitons for efficient white organic light-emitting devices," Nature, vol. 440, no. 7086, pp. 908-912, 2006.

[4] C. J. Huang, C. C. Kang, T. C. Lee, W. R. Chen, and T. H. Meen, "Improving the color purity and efficiency of blue organic light-emitting diodes (BOLED) by adding hole-blocking layer," Journal of Luminescence, vol. 129, no. 11, pp. 1292-1297, 2009.

[5] J. Kido, H. Shionota, and K. Nagai, "Single-layer white light-emitting organic electroluminescent devices based on dye-dispersed poly(N-vinylcarbazole)," Applied Physics Letters, vol. 67, no. 16, pp. 2281-2283, 1995.

[6] K. Okumoto, H. Kanno, Y. Hamada, H. Takahashi, and K. Shibata, "High efficiency red organic light-emitting devices using tetraphenyldibenzoperiflanthene-doped rubrene as an emitting layer," Applied Physics Letters, vol. 89, no. 1, Article ID 013502, 2006.

[7] G. Li and J. Shinar, "Combinatorial fabrication and studies of bright white organic light-emitting devices based on emission from rubrene-doped $4,4^{\prime}$ - bis $\left(2,2^{\prime}\right.$-diphenylvinyl)-1,1' biphenyl," Applied Physics Letters, vol. 83, no. 26, pp. 5359-5361, 2003.

[8] H. Mattoussi, H. Murata, C. D. Merritt, Y. Iizumi, J. Kido, and Z. H. Kafafi, "Photoluminescence quantum yield of pure and molecularly doped organic solid films," Journal of Applied Physics, vol. 86, no. 5, pp. 2642-2650, 1999.

[9] Y.-M. Wang, F. Teng, Z. Xu, Y.-B. Hou, S.-Y. Yang, and X.-R. $\mathrm{Xu}$, "Trap effect of an ultrathin DCJTB layer in organic lightemitting diodes," Materials Chemistry and Physics, vol. 92, no. 2-3, pp. 291-294, 2005.

[10] M. Matsumura and T. Furukawa, "Efficient electroluminescence from a rubrene sub-monolayer inserted between electron- and hole-transport layers," Japanese Journal of Applied Physics 1, vol. 40, no. 5, pp. 3211-3214, 2001.

[11] T. Li, X. Li, W. Li et al., "Tunable red emission by incorporation of a rubrene derivative in p-type and n-type hosts in organic light emitting devices," Thin Solid Films, vol. 517, no. 16, pp. 4629-4632, 2009.

[12] C.-J. Huang, T.-H. Meen, K.-C. Liao, and Y.-K. Su, “The mechanism of efficiency enhancement with proper thickness of DPVBi layer for blue organic light-emitting devices (BOLED)," Journal of Physics and Chemistry of Solids, vol. 70, no. 3-4, pp. 765-768, 2009.

[13] Q. Xue, S. Zhang, G. Xie et al., "Efficient fluorescent white organic light-emitting devices based on a ultrathin 5,6,11,12tetraphenylnaphthacene layer," Solid-State Electronics, vol. 57, no. 1, pp. 35-38, 2011.

[14] L. Li, J. Yu, X. Tang, T. Wang, W. Li, and Y. Jiang, "Efficient bright white organic light-emitting diode based on non-doped ultrathin 5,6,11,12-tetraphenylnaphthacene layer," Journal of Luminescence, vol. 128, no. 11, pp. 1783-1786, 2008.

[15] X. Tang, J. Yu, L. Li, L. Zhang, and Y. Jiang, "White organic lightemitting diodes with improved performance using phosphorescent sensitizer and ultrathin fluorescent emitter," Displays, vol. 30, no. 3, pp. 123-127, 2009.

[16] S.-H. Yang, B.-C. Hong, and S.-F. Huang, "Luminescence enhancement and emission color adjustment of white organic light-emitting diodes with quantum-well-like structures," Journal of Applied Physics, vol. 105, no. 11, Article ID 113105, 2009.

[17] Z. Ma, J. Yu, L. Li, and Y. Jiang, "Non-doped white organic lightemitting diodes consisting of three primary colors based on a bipolar emitter," Displays, vol. 33, no. 1, pp. 42-45, 2012. 

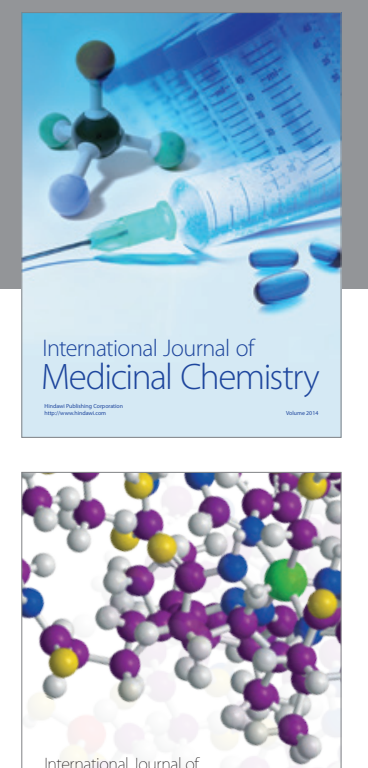

\section{Carbohydrate} Chemistry

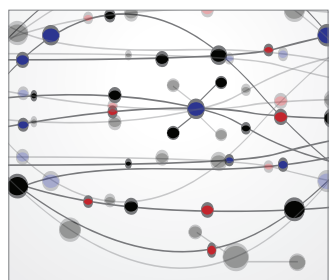

The Scientific World Journal

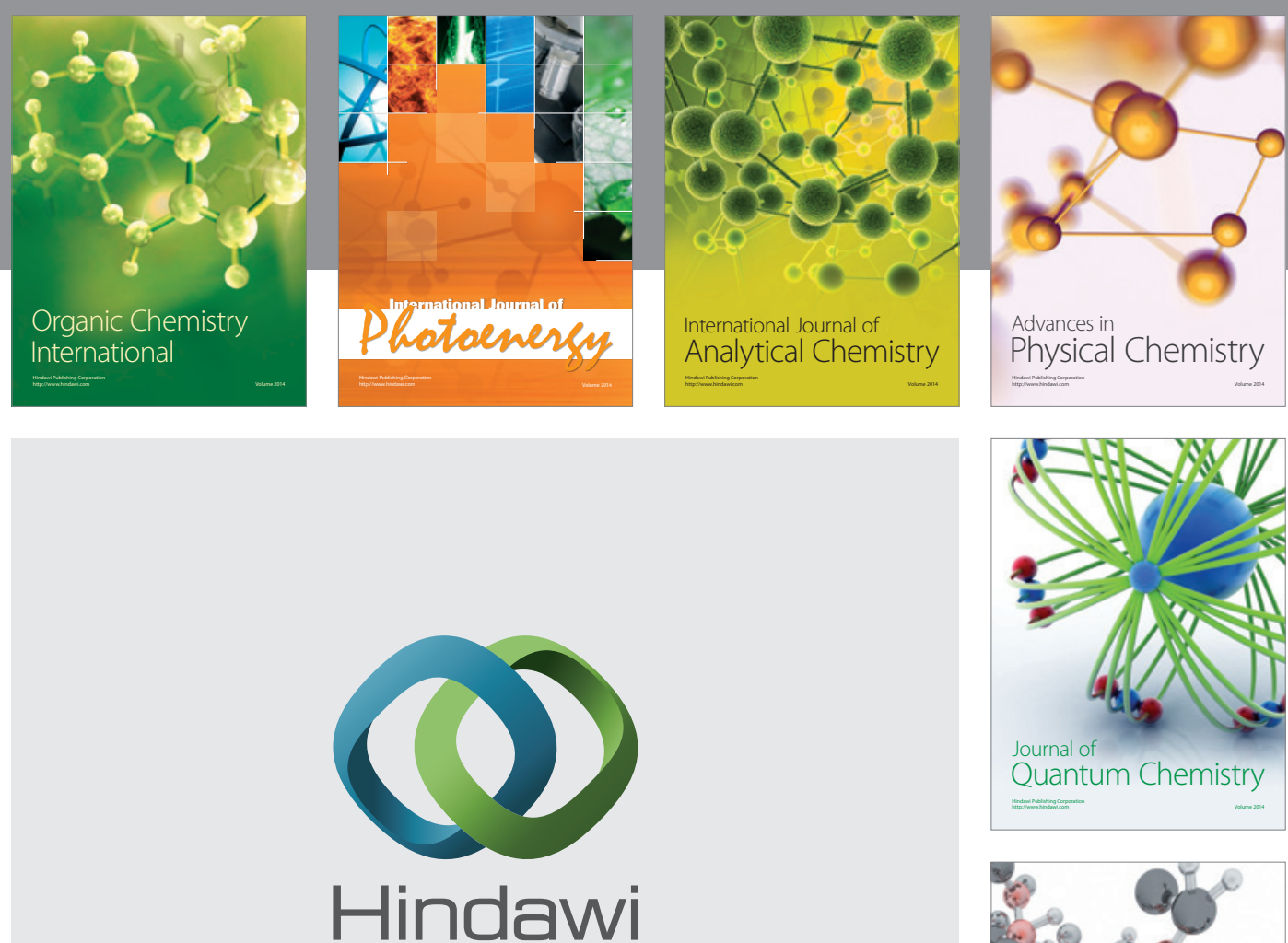

Submit your manuscripts at

http://www.hindawi.com

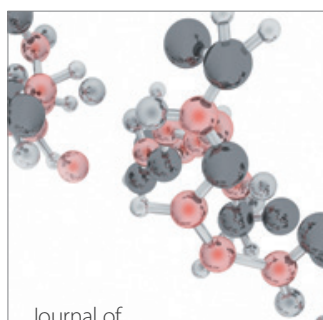

Analytical Methods

in Chemistry

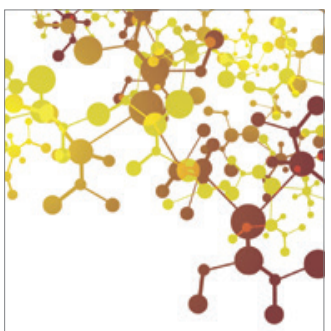

Journal of

Applied Chemistry

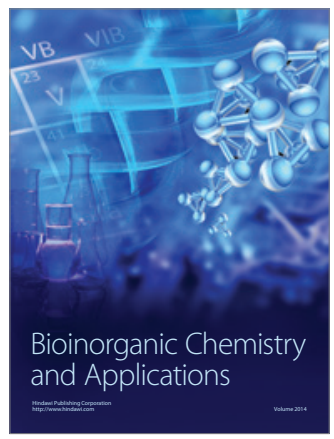

Inorganic Chemistry
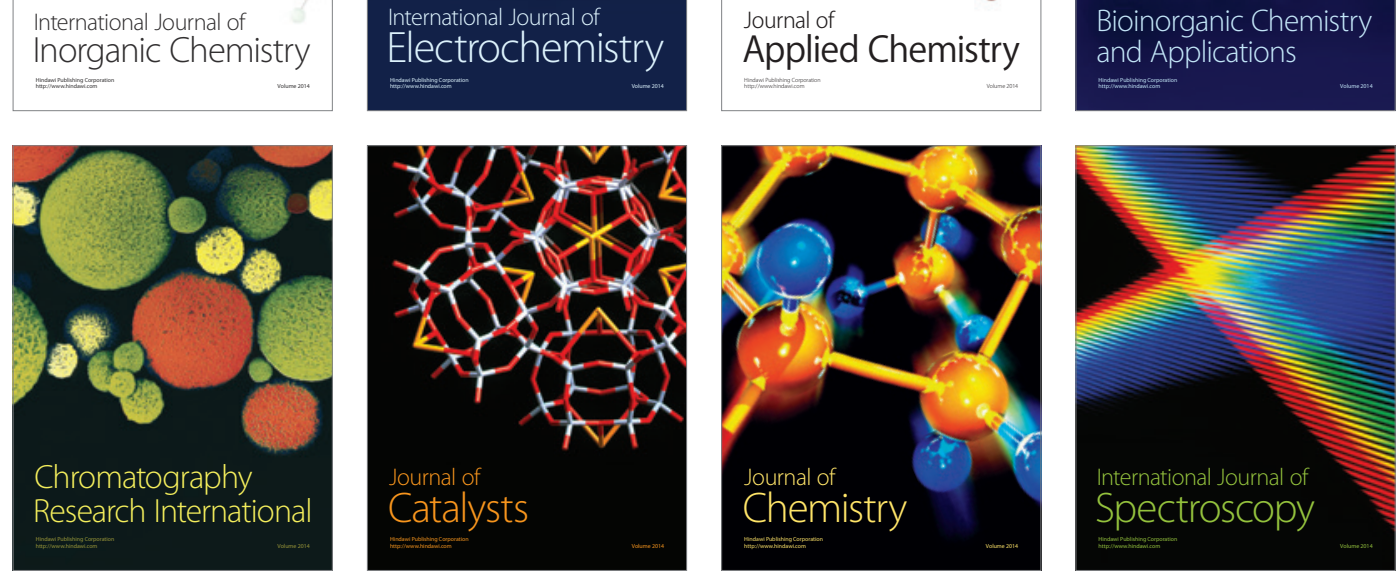